SELECCIONES MATEMÁTICAS
Universidad Nacional de Trujillo
ISSN: $2411-1783$ (Online)
Vol. $05(02): 154-163(2018)$

\title{
Existencia de solución débil para un problema no lineal con el operador p-Laplaciano fraccionario
}

\section{Existence of weak solution for a non-linear problem with fractional p-Laplacian}

\author{
Raúl Sánchez A. * and Cesar Torres L. ${ }^{\dagger}$
}

Received, Feb. 02, 2018

Accepted, Oct. 20, 2018

DOI: http://dx.doi.org/10.17268/sel.mat.2018.02.03

\begin{abstract}
Resumen
Se estudia la existencia de solución débil para un problema no lineal con el operador p-Laplaciano fraccionario para el caso donde el orden de la derivada fraccionara es $\frac{1}{p}<\alpha<1, \quad 1<q<p-1$, con $2<p<$ $\infty$, luego usando el método de minimización llamado Variedad de Nehari y su importante relación con los Fibering Maps, los cuales se definen de la forma $t \rightarrow J_{\lambda}(t u)$, donde $J_{\lambda}$ es el funcional asociado al problema no lineal a estudiar, se obtiene el resultado principal.
\end{abstract}

Palabras clave. Cálculo fraccionario, Variedad de Nehari, Fibering Maps.

\begin{abstract}
We study the existence of weak solution for a non-linear problem with fractional p-Laplacian operator for the case where the order of the fractional derivative is $\frac{1}{p}<\alpha<1, \quad 1<q<p-1$, with $2<p<\infty$, then using the minimization method called Nehari Manifold and its important relationship with the Fibering Maps, which is defined in the form $t \rightarrow J_{\lambda}(t u)$, where $J_{\lambda}$ is the functional associated to the non-linear problem to be studied, the main result is obtained.
\end{abstract}

Keywords. Fractional Calculus, Nehari Manifold, Fibering Maps

1. Introducción. La idea de generalizar la noción de derivada de orden entero para derivadas de orden no entero, surgió con el nacimiento del propio Cálculo Clásico. Fue el propio Leibniz al inventar la notación $\frac{d^{n}}{d x^{n}} f(x)$, y posiblemente por un simple deseo de jugar con los símbolos, lo que motivó, en 1965, al Marqués de L'Hôpital a preguntarle ¿Qué sucedería en el caso de ser sustituida n por $\frac{1}{2}$ ? Leibniz respondió de modo intuitivo que está aparente paradoja permitiría en el futuro extraer interesantes consecuencias [22,23]. Más adelante alcanzó una estructura matemática convincente gracias a la contribución de matemáticos de renombre como Riemann, Liouville y Abel. Fue Abel quien por primera vez aplicó el cálculo fraccionario en física, al solucionar una ecuación integral que se originó en la formulación del llamado problema de la Tautócrona y hasta el siglo XIX fue un asunto que sólo trataron algunos eminentes matemáticos, tales como Euler, Laplace, Fourier, Liouville, Riemann y Abel, entre otros [27]. Este formalismo matemático encuentra aplicaciones en áreas como viscoelasticidad, electrónica, reacciones químicas y biología [21]. Actualmente existen gran cantidad de publicaciones con aplicaciones del cálculo fraccionario en viscoelasticidad, mecánica cuántica, biología, semiconductores, propagación de ondas electromagnéticas y materiales [7, 14, 15, 17,25, 31], también se relaciona con los fenómenos de transporte por convección-difusión cuya importancia ha si sido reconocida en muchos campos de la ingeniería y las ciencias aplicadas [2], por ejemplo investigaciones importantes de vertidos de contaminantes en ríos, almacenamiento geológico profundo de residuos nucleares, problemas en biología marina, intrusión de sal marina en un estuario, problemas en ingeniería agrícola como puede ser la predicción del movimiento de pesticidas y fertilizantes a través del suelo $[1,9,10,13,18,19]$.

\footnotetext{
* Departamento Académico de Matemática. Universidad Nacional de Tumbes, Av. Arica S/N. Corresponding author rsanchez@untumbes.edu.pe.

†Departamento de Matemáticas, Universidad Nacional de Trujillo-Perú ctl_576@yahoo.es. This work is licensed under the Creative Commons Attribution-NoComercial-ShareAlike 4.0.
} 
El planteamiento de los métodos variacionales es encontrar soluciones de un determinado problema de frontera mediante la búsqueda de los puntos críticos de un funcional de energía definido en un espacio de funciones apropiadas, para luego demostrar que son soluciones débiles del problema planteado y con la teoría de la regularidad se puede obtener souciones fuertes. Torres [26] estudia la existencia de solución para un problema de Dirichlet con derivadas fraccionaria mixtas obteniendo existencia de soluciones no triviales utilizando los métodos variacionales y el teorema del paso de la montaña, asimismo, Chen et al. [3] estudian un problema de frontera con derivadas fraccionarias usando la teoría de puntos críticos y Meilan et al [16] estudian existencia de solución débil a través del método de la variedad de Nehari y el teorema Arzela-Ascoli, lo frecuente en estos estudios es reducir el problema de existencia de soluciones a uno de busqueda de puntos críticos de un funcional de energía.

Motivado por las investigaciones antes mencionadas y en particular por el estudio de Meilan at al [16], se pretende contribuir con el desarrollo de esta nueva área en la teoría de ecuaciones diferenciales fraccionarias proponiendo estudiar una ecuación diferencial con derivada fraccionaria para el caso unidimensional formulando así el siguiente problema:

$$
\begin{aligned}
{ }_{x} D_{T}^{\alpha}\left(\left|{ }_{0} D_{x}^{\alpha} u(x)\right|^{p-2}{ }_{0} D_{x}^{\alpha} u(x)\right) & =\lambda|u(x)|^{p-2} u(x)+b(x)|u(x)|^{q-1} u(x), \forall x \in[0, \Lambda] \\
u(0) & =u(\Lambda)=0
\end{aligned}
$$

Donde: $\frac{1}{p}<\alpha<1, y \quad 1<q<p-1$, con $2<p<\infty$ y $D^{\alpha}$ denota las derivada fraccionaria de Caputo [6], $b(x)$ es una función no negativa $y b \in L^{\infty}(\Omega)$.

El problema no lineal ha sido ampliamente estudiado, tanto en el caso estacionario y de evolución en el tiempo por Meilan et al [16] y un problema similar es estudiado por Drabek et al [5], Meilan et al [16] estudiaron la existencia de soluciones débiles para el caso entero del problema (1.1), y luego estudiaron el problema no estacionario con derivada temporal fraccionaria. Pero el estudio matemático de existencia de soluciones débiles para la derivada espacial fracionaria no ha sido estudiado, por lo que se abordará en éste artículo. Lo cual es importante porque permite en un próximo trabajo estudiar existencia de solución débil de la ecuación diferencial asociada al problema (1.1), que seria de tipo parobólico con variable temporal y espacial fraccionaria.

Este documento está organizado de la siguiente manera. En la sección 2 se presentan los conceptos de integral y derivada fraccionaria, espacio fraccionario, definición y propiedades la Variedad de Nehari y los Fibering Maps. En la sección 3 se demuestra el teorema obtenido como resultado principal.

\section{Preliminares.}

En esta sección, se establece las definiciones básicas del cálculo fraccionario que usaremos más adelante siguiendo el objetivo del documento.

Definición 1. Sea u una función definida en el intervalo cerrado [a,b]. La integral fraccionaria de RiemannLiouville por la derecha y la izquierda de u de orden $\alpha \in \mathbb{R}^{+}$para la función u son definidas por: ${ }_{a} I_{t}^{\alpha} u(t)=$ $\frac{1}{\Gamma(\alpha)} \int_{a}^{t}(t-s)^{\alpha-1} u(s) d s \quad y{ }_{t} I_{b}^{\alpha} u(t)=\frac{1}{\Gamma(\alpha)} \int_{t}^{b}(s-t)^{\alpha-1} u(s) d s$

Definición 2. La derivada fraccionaria de Riemann-Liouville de orden $\alpha \in \mathbb{R}^{+}$por la izquierda y derecha para la función $u$, denotadas por ${ }_{a} D_{t}^{\alpha} u(t)$ y ${ }_{t} D_{b}^{\alpha} u(t)$, respectivamente, son definidas por: ${ }_{a} D_{t}^{\alpha} u(t)=$ $\frac{d^{n}}{d t^{n}}{ }_{a} I_{t}^{n-\alpha} u(t) \quad y{ }_{t} D_{b}^{\alpha} u(t)=(-1)^{n} \frac{d^{n}}{d t^{n}}{ }_{t} I_{b}^{n-\alpha} u(t)$, sabiendo que: ${ }_{a} I_{t}^{\alpha} u(t)=\frac{1}{\Gamma(\alpha)} \int_{a}^{t}(t-s)^{\alpha-1} u(s) d s ; \quad n \in$ $\mathbb{N}, t \in[a, b]$ y $n-1 \leq \alpha<n$.

Definición 3. La derivada fraccionaria de Caputo de orden $\alpha \in \mathbb{R}^{+}$por izquierda y derecha para la función $u$, denotadas por ${ }_{a}^{c} D_{t}^{\alpha} u(t) y_{t}^{c} D_{b}^{\alpha} u(t)$, respectivamente, son definidas por

$$
\begin{aligned}
& { }_{a}^{c} D_{t}^{\alpha} u(t)={ }_{a} D_{t}^{\alpha}\left[u(t)-\sum_{k=0}^{n-1} \frac{u^{k}(a)}{k !}(t-a)^{k}\right] \quad(\text { por la izquierda }) \\
& { }_{t}^{c} D_{b}^{\alpha} u(t)={ }_{t} D_{b}^{\alpha}\left[u(t)-\sum_{k=0}^{n-1} \frac{u^{k}(b)}{k !}(b-t)^{k}\right] \quad \text { (por la derecha) }
\end{aligned}
$$

en particular, si $\alpha \in(0,1)$ se tiene ${ }_{a}^{c} D_{t}^{\alpha} u(t)={ }_{a} D_{t}^{\alpha}(u(t)-u(a)) y_{t}^{c} D_{b}^{\alpha} u(t)={ }_{t} D_{b}^{\alpha}(u(t)-u(a))$ Las derivadas fraccionarias de Caputo se definen a través de las derivadas fraccionarias Riemann-Liouville [26, 32].

Teorema 1. Sea $n \in \mathbb{N} y n-1<\alpha<n$. Si u es una función definida en $[a, b]$ para lo cual las derivadas fraccionarias de Caputo ${ }_{a}^{c} D_{t}^{\alpha} u(t)$ y ${ }_{t}^{c} D_{b}^{\alpha} u(t)$ de orden $\alpha$ ambas existen con las derivadas fraccionarias de Riemann-Liouville ${ }_{a} D_{t}^{\alpha} u(t)$ y ${ }_{t} D_{b}^{\alpha} u(t)$, entonces,

$$
{ }_{a}^{c} D_{t}^{\alpha} u(t)={ }_{a} D_{t}^{\alpha} u(t)-\sum_{k=0}^{n-1} \frac{u^{k}(a)}{\Gamma(k-\alpha+1)}(t-a)^{k} \quad, \quad t \in[a, b], \text { (por la izquierda) }
$$




$$
{ }_{t}^{c} D_{b}^{\alpha} u(t)={ }_{t} D_{b}^{\alpha} u(t)-\sum_{k=0}^{n-1} \frac{u^{k}(b)}{\Gamma(k-\alpha+1)}(b-t)^{k} \quad, \quad t \in[a, b], \text { (por la derecha) }
$$

En particular, cuando $0<\alpha<1$, se tiene

$$
\begin{gathered}
{ }_{a}^{c} D_{t}^{\alpha} u(t)={ }_{a} D_{t}^{\alpha} u(t)-\frac{u^{k}(a)}{\Gamma(k-\alpha+1)}(t-a)^{k} \quad, \quad t \in[a, b] y \\
{ }_{t}^{c} D_{b}^{\alpha} u(t)={ }_{t} D_{b}^{\alpha} u(t)-\frac{u^{k}(b)}{\Gamma(k-\alpha+1)}(b-t)^{k} \quad, \quad t \in[a, b]
\end{gathered}
$$

Demostración: Para la demostración ver [26]

Lema 1. Sea $0<\alpha \leq 1,1 \leq p<\infty$. Para cualquier $u \in L^{p}[0, T]$, se tiene

$$
\left\|{ }_{0} I_{\xi}^{\alpha} u\right\|_{L^{p}[0, t]} \leq \frac{t^{\alpha}}{\Gamma(\alpha+1)}\|u\|_{L^{p}[0, t]}, \text { para todo } \xi \in[0, t], t \in[0, T]
$$

Demostración: Ver demostración en [26].

Teorema 2. Propiedades de la integral fraccionaria de Rimmann-Liouville.

(1) Tenemos ${ }_{a} I_{t}^{\alpha}\left({ }_{a} I_{t}^{\beta}\right)={ }_{a} I_{t}^{\alpha+\beta} y_{t} I_{b}^{\alpha}\left({ }_{t} I_{b}^{\beta}\right)={ }_{t} I_{b}^{\alpha+\beta}, \quad \forall \alpha, \beta>0$

(2) La inversa a izquierda. Sea $u \in L^{1}[a, b]$ y $\alpha>0$,

$$
{ }_{a} D_{t}^{\alpha}\left({ }_{a} I_{t}^{\alpha} u(t)\right)=u(t), \quad \text { c.t.p. } \quad t \in[a, b] \quad y{ }_{t} D_{b}^{\alpha}\left({ }_{t} I_{b}^{\alpha} u(t)\right) \quad=u(t), \quad \text { c.t.p. } t \in[a, b] .
$$

(3) Para $n-1<\alpha<n$, si las derivadas fraccionarias de Riemann-Liouville ${ }_{a} D_{t}^{\alpha} u(t)$ y ${ }_{t} D_{b}^{\alpha} u(t)$, de la función u son integrables sobre $[a, b]$, entonces

$$
\begin{aligned}
{ }_{a} I_{t}^{\alpha}\left({ }_{a} D_{t}^{\alpha} u(t)\right) & =u(t)-\sum_{k=1}^{n}\left[{ }_{a} I_{t}^{k-\alpha} u(t)\right]_{t=a} \frac{(t-a)^{\alpha-k}}{\Gamma(\alpha-k+1)}, \\
{ }_{t} I_{b}^{\alpha}\left({ }_{t} D_{b}^{\alpha} u(t)\right) & =u(t)-\sum_{k=1}^{n}\left[{ }_{t} I_{b}^{k-\alpha} u(t)\right]_{t=b} \frac{(-1)^{n}(b-t)^{\alpha-k}}{\Gamma(\alpha-k+1)},
\end{aligned}
$$

para $t \in[a, b]$

(4) Integración por partes: $\int_{a}^{b}\left[{ }_{a} I_{t}^{\alpha} u(t)\right] v(t) d t=\int_{a}^{b} u(t){ }_{t} I_{b}^{\alpha} v(t) d t, \quad \alpha>0$, siempre que $u \in L^{p}[a, b], v \in L^{q}[a, b]$ y $p \geq 1, q \geq 1 y \frac{1}{p}+\frac{1}{q}<1+\alpha o ́ p \neq 1, q \neq 1 y \frac{1}{p}+\frac{1}{q}=1+\alpha$

$$
\int_{a}^{b}\left[{ }_{a} D_{t}^{\alpha} u(t)\right] v(t) d t=\int_{a}^{b} u(t){ }_{t} D_{b}^{\alpha} v(t) d t, \quad 0<\alpha \leq 1,
$$

siempre que las condiciones: $u(a)=u(b)=0, \quad u^{\prime} \in L^{\infty}[a, b], \quad v \in L^{1}[a, b]$ ó $u(a)=u(b)=0, \quad v^{\prime} \in L^{\infty}[a, b], \quad u \in L^{1}[a, b]$, se cumplan.

(5) Sea $0<\frac{1}{p}<\alpha \leq 1$ y $u(x) \in L^{P}[a, b]$, entonces ${ }_{0} I_{t}^{\alpha} u(t)$ es Holder continua sobre $[0, T]$ con exponente $\alpha-\frac{1}{p} y \lim _{t \rightarrow 0^{+}}{ }_{0} I_{t}^{\alpha} u(t)=0$. Consecuentemente, ${ }_{0} I_{t}^{\alpha} u(t)$ puede ser extendido continuamente por 0 en $x=0$.

Demostración: Para la demostración ver [32]

2.1. Espacio de derivadas fraccionarias. Se considera el Lema 1 y resultados de [26, 32]. Para cualquier $t \in[0, T] y 1 \leq p<\infty,\|u\|_{L^{p}[0, t]}=\left(\int_{0}^{t}|u(s)|^{p} d s\right)^{\frac{1}{p}},\|u\|_{L^{p}}=\left(\int_{0}^{T}|u(s)|^{p} d s\right)^{\frac{1}{p}} y\|u\|_{\infty}=\operatorname{máx}_{t \in[0, T]}|u(t)|$.

Definición 4. Sea $0<\alpha \leq 1$ y $1<p<\infty$. El espacio de derivadas fraccionarias $E_{0}^{\alpha, p}$ es definido por $E_{0}^{\alpha, p}=\left\{u \in L^{p}[0, T]:{ }_{0} D_{t}^{\alpha} u \in L^{p}[0, T], u(0)=u(T)=0\right\}={\overline{C_{0}^{\infty}[0, T]}}^{\|\cdot\|_{\alpha, p}}$. Donde $\|u\|_{\alpha, p}$ es definida por

$$
\|u\|_{\alpha, p}^{p}=\int_{0}^{T}|u(t)|^{p} d t+\int_{0}^{T}\left|{ }_{0} D_{t}^{\alpha} u(t)\right|^{p} d t, \quad \forall u \in E_{0}^{\alpha, p}
$$

Observación 1. Para cualquier $u \in E_{0}^{\alpha, p}$, observando el hecho de que $u(0)=0$, tenemos ${ }_{a}^{c} D_{t}^{\alpha} u(t)={ }_{a} D_{t}^{\alpha} u(t), t \in$ $[0, T]$, según Teorema 1.

Demostración: Para la demostración ver [32]. 
Proposición 1. Sea $0<\alpha \leq 1$ y $1 \leq p<\infty$. El espacio derivado fraccionario $E_{0}^{\alpha, p}$ es un espacio de Banach, separable y reflexivo.

Demostración: Para la demostración ver [11].

Lo siguiente son propiedades del espacio fraccionario $E_{0}^{\alpha, p}$.

Lema 2. Sea $0<\alpha \leq 1$ y $1 \leq p<\infty$. Para cualquier $u \in L^{p}[0, T]$ tenemos

$$
\left\|_{0} I_{\xi}^{\alpha} u\right\|_{L^{p}[0, t]} \leq \frac{t^{\alpha}}{\Gamma(\alpha+1)}\|u\|_{L^{p}[0, t]}, \text { para } \xi \in[0, t], t \in[0, T] .
$$

Demostración: Para la demostración ver [11].

Proposición 2. Sea $0<\alpha \leq 1$ y $1 \leq p<\infty$. Para todo $u \in E_{0}^{\alpha, p}$, tenemos

$$
\|u\|_{L^{p}} \leq \frac{T^{\alpha}}{\Gamma(\alpha+1)}\left\|_{0} D_{t}^{\alpha} u\right\|_{L^{p}}
$$

Si $\alpha>1$ y $\frac{1}{p}+\frac{1}{q}=1$, entonces

$$
\|u\|_{\infty} \leq \frac{T^{\alpha-1 / p}}{\Gamma(\alpha)((\alpha-1) q+1)^{1 / q}}\left\|_{0} D_{t}^{\alpha} u\right\|_{L^{p}}
$$

Demostración: Para la demostración ver [12].

Observación 2. De acuerdo a la Proposición 2 se tiene que la norma $\|\cdot\|_{E_{0}^{\alpha, p}}$ del espacio fraccionario $E_{0}^{\alpha, p}$ es equivalente a la norma $\left\|_{0} D_{t}^{\alpha} u(t)\right\|_{L^{p}}$. Esto es $\|u\|_{E_{0}^{\alpha, p}}=\left\|_{0} D_{t}^{\alpha} u(t)\right\|_{L^{p}}=\left(\int_{0}^{t}\left|{ }_{0} D_{t}^{\alpha} u(t)\right|^{p} d t\right)^{1 / p}$. Demostración: Para la demostración ver [28] .

A partir de ahora denotamos $\|\cdot\|_{E_{0}^{\alpha, p}}$ como $\|.\|_{\alpha, p}$.

Observación 3. Por otra parte de (2.5) tenemos

$$
\|u\|_{\infty} \leq \frac{T^{\alpha-1 / \alpha}}{\Gamma(\alpha)((\alpha-1) q+1)^{1 / q}}\left\|_{0} D_{t}^{\alpha} u(t)\right\|_{L^{p}} \leq \frac{T^{\alpha-1 / \alpha}}{\Gamma(\alpha)((\alpha-1) q+1)^{1 / q}}\left\|_{0} D_{t}^{\alpha} u(t)\right\|_{\alpha, p}
$$

es decir $E_{0}^{\alpha, p}$ está inyectado continuamente en $C[0, T]$ para $\alpha>\frac{1}{p}$.

Demostración: Para la demostración ver [32].

Proposición 3. Sea $0<\alpha \leq 1$ y $1 \leq p<\infty$. Asumamos que $\alpha>\frac{1}{p}$ y la sucesión $\left\{u_{k}\right\}$ converge débilmente a u en $E_{0}^{\alpha, p}$, ie, $u_{k} \rightarrow u$. Entonces $u_{k} \rightarrow$ en $C[0, T]$, ie, $\left\|u_{k}-u\right\|_{\infty} \rightarrow 0, k \rightarrow \infty$.

Demostración: Para la demostración ver [12].

Teorema 3. Sea $\alpha \in\langle 0,1\rangle$, entonces la inyección continua $E_{0}^{\alpha, p} \hookrightarrow L^{p}[0, T]$ es compacta. Demostración: Para la demostración ver [26]. $\square$

2.2. Problema No Lineal. Consideremos el problema:

$$
\begin{aligned}
{ }_{x} D_{T}^{\alpha}\left(\left|{ }_{0} D_{x}^{\alpha} u(x)\right|^{p-2}{ }_{0} D_{x}^{\alpha} u(x)\right) & =\lambda|u(x)|^{p-2} u(x)+b(x)|u(x)|^{q-1} u(x), \forall x \in[0, \Lambda] \\
u(0) & =u(\Lambda)=0
\end{aligned}
$$

Donde: $\frac{1}{p}<\alpha<1, y \quad 1<q<p-1$, con $2<p<\infty$ Así para cada $u \in E_{0}^{\alpha, p}$, el problema (2.6) tiene como funcional asociado

$$
J_{\lambda}(u)=\frac{1}{p} \int_{[0, \Lambda]}\left|{ }_{0} D_{x}^{\alpha} u(x)\right|^{p} d x-\frac{\lambda}{p} \int_{[0, \Lambda]}|u(x)|^{p} d x-\frac{1}{q+1} \int_{[0, \Lambda]} b(x)|u|^{q+1} d x
$$

Diremos que $u \in E_{0}^{\alpha, p}$ es solución débil del problema (2.6) si

$$
J_{\lambda}^{\prime}(u) v=\int_{[0, \Lambda]}\left|{ }_{0} D_{x}^{\alpha} u\right|_{0}^{p-2} D_{x}^{\alpha} u_{0} D_{x}^{\alpha} v d x-\lambda \int_{[0, \Lambda]}|u|^{p-2} u v d x-\int_{[0, \Lambda]} b(x)|u|^{q-1} u v d x=0
$$

para todo $v \in E_{0}^{\alpha, p}$.

Consideremos el problema de autovalores asociado al operador p-Laplaciano fraccionario dado por

$$
\left\{\begin{array}{c}
{ }_{x} D_{\Lambda}^{\alpha}\left(\left|{ }_{0} D_{x}^{\alpha} u(x)\right|^{p-2}{ }_{0} D_{x}^{\alpha} u(x)\right)=\lambda|u(x)|^{p-2} u(x), \text { para } x \in[0, \Lambda] \\
u(0)=u(\Lambda)=0,
\end{array}\right.
$$

$$
\text { Note que, } \quad \lambda_{1}=\min _{u \in E_{0}^{\alpha, p}} \frac{\int_{0}^{\Lambda}\left|{ }_{0} D_{x}^{\alpha} u(x)\right|^{p} d x}{\int_{0}^{\Lambda}|u(x)|^{p} d x}, \quad u \neq 0 . \quad \text { Ver [29] }
$$

Lema 3. 
(i) Suponga que $\lambda<\lambda_{1}$; entonces $J_{\lambda}$ es acotado inferiormente en $E_{0}^{\alpha, p}$.

(ii) Si $\lambda>\lambda_{1}$, entonces $J_{\lambda}$ no es acotado inferiormente.

Demostración:

(i) De acuerdo con (2.9) se tiene que: $\lambda_{1} \int_{[0, \Lambda]}|u(x)|^{p} d x \leqslant \int_{[0, \Lambda]}\left|{ }_{0} D_{x}^{\alpha} u(x)\right|^{p} d x$

$$
\begin{gathered}
\text { luego, } \int_{[0, \Lambda]}{ }_{0} D_{x}^{\alpha}|u(x)|^{p} d x-\lambda \int_{[0, \Lambda]}|u(x)|^{p} d x \geqslant\left(\lambda_{1}-\lambda\right) \int_{[0, \Lambda]}|u(x)|^{p} d x \\
J_{\lambda}(u) \geq \frac{\left(\lambda_{1}-\lambda\right)}{p} \int_{[0, \Lambda]}|u(x)|^{p} d x-\frac{1}{q+1} \int_{[0, \Lambda]} b(x)|u(x)|^{q+1} d x
\end{gathered}
$$

Como $b \in L^{\infty}$ se tiene que $J_{\lambda}(u) \geq \frac{1}{p}\left(\lambda_{1}-\lambda\right) \int_{[0, \Lambda]}|u(x)|^{p} d x-\frac{\bar{b}}{q+1} \int_{[0, \Lambda]}|u(x)|^{q+1} d x$, luego aplicando desigualdad de holder sabiendo que $1<q<p-1$ y $\frac{2}{p}<\frac{q+1}{p}<1$ y $1-\left(\frac{q+1}{p}\right)>0$, se obtiene que

$$
J_{\lambda}(u) \geq \frac{1}{p}\left(\lambda_{1}-\lambda\right) \int|u(x)|^{p} d x-\frac{\bar{b}}{q+1}|[0, \Lambda]|^{1-(q+1) / p}\left(\int|u(x)|^{p} d x\right)^{(q+1) / p}
$$

Por lo tanto, $J_{\lambda}$ es acotado inferiormente en $E_{0}^{\alpha, p}$ cuando $\lambda<\lambda_{1}$.

(ii) Ahora si $\lambda>\lambda_{1}$. Sea $\phi$ la eigenfunción asociada al autovalor principal $\lambda_{1}$, esto es $\left(\phi, \lambda_{1}\right)$ es una solución de (2.8) y $u \neq 0$, entonces

$$
\lambda_{1}=\frac{\int_{0}^{\Lambda}\left|{ }_{0} D_{x}^{\alpha} \phi(x)\right|^{p} d x}{\int_{0}^{\Lambda}|\phi(x)|^{p} d x},
$$

Procediento de igual forma que $(i)$ y tomando el límite a $J_{\lambda}\left(t \phi_{1}\right)$ cuando $t \rightarrow \infty$ se tiene

$$
\lim _{t \rightarrow \infty} J_{\lambda}\left(t \phi_{1}\right)=\lim _{t \rightarrow \infty}|t|^{p}\left[\frac{\lambda_{1}}{p} \int_{[0, \Lambda]}\left|\phi_{1}\right|^{p} d x-\frac{\lambda}{p} \int_{[0, \Lambda]}\left|\phi_{1}\right|^{p} d x-\frac{1}{(q+1) t^{p-(q+1)}} \int_{[0, \Lambda]} b(x)\left|\phi_{1}\right|^{q+1} d x\right]
$$

se obtiene que $\lim _{t \rightarrow \infty} J_{\lambda}\left(t \phi_{1}\right)=-\infty$, por lo tanto $J_{\lambda}$ no es acotado inferiormente en $E_{0}^{\alpha, p}$.

2.3. Variedad de Nehari. Consideremos el siguiente subconjunto de $E_{0}^{\alpha, p}$

$$
N_{\lambda}=\left\{u \in E_{0}^{\alpha, p}:\left\langle J_{\lambda}^{\prime}(u), u\right\rangle=0, u \neq 0\right\}
$$

denominado Variedad de Nehari, donde $\langle, \quad\rangle$ denota la dualidad usual. Observe que si u es un punto crítico del funcional $J_{\lambda}$, esto es $J^{\prime}{ }_{\lambda}(u) v=0$ para todo $v \in E_{0}^{\alpha, p}$ entonces $u \in N_{\lambda}$. Así, diremos que $u \in N_{\lambda}$ si y solo si satisface $\int_{[0, \Lambda]}\left|{ }_{0} D_{x}^{\alpha} u(x)\right|^{p} d x-\lambda \int_{[0, \Lambda]}|u(x)|^{p} d x-\int_{[0, \Lambda]} b(x)|u|^{q+1} d x=0$

Proposición 4. [4] El conjunto $N_{\lambda}$ es no vacío y es una subvariedad de $E_{0}^{\alpha, p}$.

2.4. Operador Fibering Maps. Consideremos las funciones reales de variable positiva de la forma: $\phi_{u}(t)$ : $t \rightarrow J_{\lambda}(t u)(t>0)$, estas funciones se conoce con el nombre de Fibering Maps y son denominados asi por Drabek y Pohozaev [5]. Así para cada $u \in E_{0}^{\alpha, p}$ se tiene

$$
\begin{gathered}
\phi_{u}(t)=J_{\lambda}(t u)=\frac{t^{p}}{p} \int_{[0, \Lambda]}\left(\left|{ }_{0} D_{x}^{\alpha} u\right|^{p}-\lambda|u|^{p}\right) d x-\frac{t^{q+1}}{q+1} \int_{[0, \Lambda]} b|u|^{q+1} d x \\
\phi_{u}^{\prime}(t)=t^{p-1} \int_{[0, \Lambda]}\left(\left|{ }_{0} D_{x}^{\alpha} u(x)\right|^{p}-\lambda|u(x)|^{p}\right) d x-t^{q} \int_{[0, \Lambda]} b(x)|u(x)|^{q+1} d x \\
\phi_{u}^{\prime \prime}(t)=(p-1) t^{p-2} \int_{[0, \Lambda]}\left(\left|{ }_{0} D_{x}^{\alpha} u(x)\right|^{p}-\lambda|u(x)|^{p}\right) d x-q t^{q-1} \int_{[0, \Lambda]} b(x)|u(x)|^{q+1} d x
\end{gathered}
$$

El siguiente lema es decisivo en la relación entre la Variedad de Nehari y los Fibering Maps, pues garantiza una correspondencia entre los puntos críticos de $\phi_{u}$ y los elementos de $N_{\lambda}$.

Lema 4. Sea $\phi_{u}$ el operador definido en (2.11), con con $t>0$ y sea $u \in E_{0}^{\alpha, p}$, entonces:

(i) $u \in N_{\lambda}$ si y solo si $\phi_{u}^{\prime}(1)=0$ 
(ii) Mas generalmente tu $\in N_{\lambda}$ si y solo si $\phi_{u}^{\prime}(t)=0$

Demostración:

(i) $\phi_{u}^{\prime}(1)=\int_{[0, \Lambda]}\left|{ }_{0} D_{x}^{\alpha} u(x)\right|^{p} d x-\lambda \int_{[0, \Lambda]}|u(x)|^{p} d x-\int_{[0, \Lambda]} b(x)|u|^{q+1} d x=J_{\lambda}^{\prime}(u) u$

(ii) Veamos primero $(\Leftarrow)$

$$
\begin{gathered}
0=\phi_{u}^{\prime}(t)=t^{p-1} \int_{[0, \Lambda]}\left(\left|{ }_{0} D_{x}^{\alpha} u(x)\right|^{p}-\lambda|u(x)|^{p}\right) d x-t^{q} \int_{[0, \Lambda]} b(x)|u(x)|^{q+1} d x \\
0=t^{p} \int_{[0, \Lambda]}\left(\left|{ }_{0} D_{x}^{\alpha} u(x)\right|^{p}-\lambda|u(x)|^{p}\right) d x-t^{q+1} \int_{[0, \Lambda]} b(x)|u(x)|^{q+1} d x=J_{\lambda}^{\prime}(t u) t u
\end{gathered}
$$

$(\Rightarrow)$ Como tu $\in N_{\lambda}$ se tiene

$$
\begin{gathered}
0=J_{\lambda}^{\prime}(t u) t u=t^{p} \int_{[0, \Lambda]}\left(\left|{ }_{0} D_{x}^{\alpha} u(x)\right|^{p}-\lambda|u(x)|^{p}\right) d x-t^{q+1} \int_{[0, \Lambda]} b(x)|u(x)|^{q+1} d x \\
0=J_{\lambda}^{\prime}(t u) t u=t^{p-1} \int_{[0, \Lambda]}\left(\left|{ }_{0} D_{x}^{\alpha} u(x)\right|^{p}-\lambda|u(x)|^{p}\right) d x-t^{q} \int_{[0, \Lambda]} b(x)|u(x)|^{q+1} d x=\phi_{u}^{\prime}(t), \text { con } t>0
\end{gathered}
$$

De esta forma, se sabe que $u \in N_{\lambda}$, si y solo si $\phi_{u}^{\prime}(1)=0$ y es natural dividir la Variedade de Nehari en tres conjuntos disjuntos. Note que, de (2.12) y (2.13), se tiene

$$
\phi_{u}^{\prime \prime}(t)=t^{q-1}[(p-1)-q] \int_{[0, \Lambda]} b(x)|u(x)|^{q+1} d x
$$

$y$ como $t>0$ y $1<q<p-1$, se tiene, $\phi_{u}^{\prime \prime}(t)>0 \Leftrightarrow \int_{[0, \Lambda]} b(x)|u(x)|^{q+1} d x>0$

$$
\phi_{u}^{\prime \prime}(t)<0 \Leftrightarrow \int_{[0, \Lambda]} b(x)|u(x)|^{q+1} d x<0, y \phi_{u}^{\prime \prime}(t)=0 \Leftrightarrow \int_{[0, \Lambda]} b(x)|u(x)|^{q+1} d x=0
$$

Se puede entonces definir de $N_{\lambda}$ tres conjuntos como: $N_{\lambda}^{+}=\left\{u \in N_{\lambda}: \int b(x)|u(x)|^{q+1} d x>0\right\}$

$$
N_{\lambda}^{-}=\left\{u \in N_{\lambda}: \int b(x)|u(x)|^{q+1} d x<0\right\} y N_{\lambda}^{0}=\left\{u \in N_{\lambda}: \int b(x)|u(x)|^{q+1} d x=0\right\}
$$

$N_{\lambda}^{+}, N_{\lambda}^{-}$y $N_{\lambda}^{0}$ que corresponde a los puntos de mínimo local, máximo local y de inflexión de $\phi_{u}$ respectivamente.

El análisis del comportamiento de $\phi_{u}$ es en relación al signo de:

$$
R(u)=\int_{[0, \Lambda]}\left(\left|{ }_{0} D_{x}^{\alpha} u(x)\right|^{p}-\lambda|u(x)|^{p}\right) d x \text { y } B(u)=\int_{[0, \Lambda]} b(x)|u(x)|^{q+1} d x .
$$

Sea $u \in E_{0}^{\alpha, p}$ y si $\phi_{u}^{\prime}(t)=0$ se concluye que:

(i) Si $R(u)$ y $B(u)$, tienen el mismo signo, entonces $\phi_{u}$ tiene un único punto crítico, luego se despeja t:

$$
t_{u}=\left[\frac{\int_{[0, \Lambda]} b(x)|u(x)|^{q+1} d x}{\int_{[0, \Lambda]}\left(\left|{ }_{0} D_{x}^{\alpha} u(x)\right|^{p}-\lambda|u(x)|^{p}\right) d x}\right]^{\frac{1}{(p-1-q)}}
$$

Así, este punto crítico es un mínimo cuando $t_{u} \in N_{\lambda}^{+}$si y solo si $B(u)>0$ y es es un máximo cuando $t_{u} \in N_{\lambda}^{-}$si y solo si $B(u)<0$.

(ii) Si $R(u)$ y $B(u)$, tienen signos opuestos, entonces $\phi_{u}$ no tiene puntos de inflexión, por esta razón no hay múltiplos de $u$ en $N_{\lambda}$.

Por lo tanto si definimos: $L_{+}(\lambda)=\left\{u \in E_{0}^{\alpha, p}:\|u\|=1, \int_{[0, \Lambda]}\left(\left|{ }_{0} D_{x}^{\alpha} u(x)\right|^{p}-\lambda|u(x)|^{p}\right) d x>0\right\}$ $B_{+}=\left\{u \in E_{0}^{\alpha, p}:\|u\|=1, \int_{[0, \Lambda]} b(x)|u(x)|^{q+1} d x>0\right\}$. Análogamente, se define $L_{-}(\lambda), L_{0}(\lambda), B_{-} y B_{0}$ al reemplazar " $>0$ " por “ $<0 ” " ~ "=0 ”$. Según sea apropiado, se tiene lo siguiente:

(i) Si $u \in L_{+}(\lambda) \cap B_{+}$, entonces $t \rightarrow \phi_{u}(t)$ tiene un mínimo local $t=t_{u} y t_{u} u \in N_{\lambda}^{+}$.

(ii) Si $u \in L_{-}(\lambda) \cap B_{-}$, entonces $t \rightarrow \phi_{u}(t)$ tiene un máximo local $t=t_{u} y t_{u} u \in N_{\lambda}^{-}$.

(iii) Si $u \in L_{+}(\lambda) \cap B_{-}$, entonces $t \rightarrow \phi_{u}(t)$ es estrictamente creciente y ningún múltiplo de u esta en $N_{\lambda}$. 
(iv) Si $u \in L_{-}(\lambda) \cap B_{+}$, entonces $t \rightarrow \phi_{u}(t)$ es estrictamente decreciente y ningún múltiplo de u esta en $N_{\lambda}$. El siguiente lema garantiza que un punto crítico, $u \in N_{\lambda}$, del funcional $J_{\lambda}$ restringido a la Variedad de Nehari tal que $u \notin N_{\lambda}^{0}$ es en realidad un punto crítico en $E_{0}^{\alpha, p}$.

Lema 5. Suponga que $u_{0} \in N_{\lambda}$ es un punto local máximo o punto local mínimo para $J_{\lambda}$ en $N_{\lambda}$, y que $u_{0} \notin N_{\lambda}^{0}$; entonces $u_{0}$ es un punto crítico de $J_{\lambda}$, es decir $J_{\lambda}^{\prime}\left(u_{0}\right)=0$ en $\left(E_{0}^{\alpha, p}\right)^{*}$. Demostración: Si $u_{0}$ es un punto local minimizante para $J_{\lambda}$ en $N_{\lambda}$, entonces $u_{0}$ es una solución de: Minimize $J_{\lambda}(u)$ sujeto a $r_{\lambda}(u)=0$, donde $r_{\lambda}(u)=\int_{[0, \Lambda]}\left(\left|{ }_{0} D_{x}^{\alpha} u(x)\right|^{p}-\lambda|u(x)|^{p}-b(x) u(x)^{q+1}\right) d x$

de ahí, por la teoría de multiplicadores de Lagrange, existe $\mu \in \mathbb{R}$ tal que

$$
J_{\lambda}^{\prime}\left(u_{0}\right)=\mu r_{\lambda}^{\prime}\left(u_{0}\right) \text {, en }\left(E_{0}^{\alpha, p}\right)^{*} .
$$

De esta manera,

$$
\left\langle J_{\lambda}^{\prime}\left(u_{0}\right), u_{0}\right\rangle_{E_{0}^{\alpha, p}}=\mu\left\langle r_{\lambda}^{\prime}\left(u_{0}\right), u_{0}\right\rangle_{E_{0}^{\alpha, p}}
$$

puesto que $u_{0} \in N_{\lambda}$, se tiene $\left\langle J_{\lambda}^{\prime}\left(u_{0}\right), u_{0}\right\rangle=0 y$

$$
\begin{aligned}
\left\langle r_{\lambda}^{\prime}\left(u_{0}\right), u_{0}\right\rangle_{E_{0}^{\alpha, p}}=p & {\left[\int_{[0, \Lambda]}\left(\left|{ }_{0} D_{x}^{\alpha} u_{0}(x)\right|^{p}-\lambda\left|u_{0}(x)\right|^{p}\right) d x\right]-(q+1) \int_{[\Lambda, p]} b(x)\left|u_{0}(x)\right|^{q+1} d x } \\
& \left\langle r_{\lambda}^{\prime}\left(u_{0}\right), u_{0}\right\rangle_{E_{0}^{\alpha, p}}=(p-q-1)\left[\int_{[\Lambda, p]} b(x)\left|u_{0}(x)\right|^{q+1}\right]
\end{aligned}
$$

luego, si $u_{0} \notin N_{\lambda}^{0}, y\left\langle r_{\lambda}^{\prime}\left(u_{0}\right), u_{0}\right\rangle_{X} \neq 0$ y por (2.15) la única posibilidad es $\mu=0$. Esto completa la prueba.

2.5. Propiedades de la Variedad de Nehari. En esta sección, vamos a discutir el rol fundamental que desempeña la condición $L_{-}(\lambda) \subseteq B_{-}$en la determinación de la naturaleza de la Variedad de Nehari. Cuando $\lambda<\lambda_{1}$, $\int_{[0, \Lambda]}\left(\left|{ }_{0} D_{x}^{\alpha} u(x)\right|^{p}-\lambda|u(x)|^{p}\right) d x>0$, para todo $u \in E_{0}^{\alpha, p}$ y $L_{+}(\lambda)=\left\{u \in E_{0}^{\alpha, p}:\|u\|=1\right\} y L_{-}(\lambda)=\emptyset$ , $L_{0}(\lambda)=\emptyset$, cuando $\lambda=\lambda_{1}$, se tiene $L_{-}(\lambda)=\emptyset, L_{0}(\lambda)=\left\{\phi_{1}\right\}$ y cuando $\lambda>\lambda_{1}, L_{-}(\lambda)$ se convierte en no vacio y se hace cada vez más grande a medida que $\lambda$ aumenta.

Teorema 4. Suponga que existe $\hat{\lambda}$ tal que, para todo $\lambda<\hat{\lambda}, L_{-}(\lambda) \subseteq B_{-}$. Entonces, $\forall \lambda<\hat{\lambda}$, se cumple que

(i) $L_{0}(\lambda) \subseteq B_{-}$y así $L_{0}(\lambda) \cap B_{0}=\emptyset$.

(ii) $N_{\lambda}^{+}$, es acotado.

(iii) $0 \notin \overline{N_{\lambda}^{-}}, y N_{\lambda}^{-}$es cerrado.

(iv) $\overline{N_{\lambda}^{+}} \cap N_{\lambda}^{-}=\emptyset$.

Demostración: Para la demostración revisar [16]

Teorema 5. Suponga que existe $\hat{\lambda}$ tal que, para todo $\lambda<\hat{\lambda}, L_{-}(\lambda) \subseteq B_{-}$. Entonces, $\forall \lambda<\hat{\lambda}$, se cumple que

(i) $J_{\lambda}$ es acotado inferiormente en $N_{\lambda}^{+}$

(ii) $\inf _{u \in N_{\lambda}^{-}} J_{\lambda}(u)>0$, a condición de que $N_{\lambda}^{-}$es no vacio.

Demostración: Para la demostración revisar [16]

\section{Resultado.}

Teorema 6. Supongamos que $L_{-}(\lambda) \subseteq B_{-}(\lambda)$ para todo $\lambda<\hat{\lambda}$; entonces $\forall \lambda<\hat{\lambda}$

(i) Existe un punto minimizante para $J_{\lambda}$ en $N_{\lambda}^{+}$

(ii) Existe un punto minimizante para $J_{\lambda}$ en $N_{\lambda}^{-}$siempre que $L_{-}(\lambda)$ es no vacio.

Demostración:

(i) Por el Teorema 5, $J_{\lambda}$ es acotado inferiormente en $N_{\lambda}^{+}$, por definición de ínfimo, existe $\left\{u_{n}\right\} \subseteq N_{\lambda}^{+}$una sucesión minimizante tal que $\lim _{n \rightarrow \infty} J_{\lambda}\left(u_{n}\right)=\inf _{u \in N_{\lambda}^{+}} J_{\lambda}(u)<0$.

Como, $J_{\lambda}\left(u_{n}\right)=\left(\frac{1}{p}-\frac{1}{q+1}\right) \int_{[0, \Lambda]}^{n \rightarrow \infty} b(x)\left|u_{n}\right|^{q+1} d x$, con $\left(\frac{1}{p}-\frac{1}{q+1}\right)<0 y \int_{[0, \Lambda]} b(x)\left|v_{0}\right|^{q+1} d x>0$ para todo $n$, se tiene $J_{\lambda}\left(u_{n}\right)<0$, además, por la parte (ii) del Teorema $5, N_{\lambda}^{+}$es acotado, de ahí suponga $u_{n} \rightarrow u_{0}$ en $E_{0}^{\alpha, p}$ y $u_{n} \rightarrow u_{0}$ en $L^{q+1}([0, \Lambda])$.

Luego $\int_{[0, \Lambda]} b\left|u_{0}(x)\right|^{q+1} d x=\lim _{n \rightarrow \infty} \int_{[0, \Lambda]} b\left|u_{n}(x)\right|^{q+1} d x>0$ y así $\frac{u_{0}}{\left\|u_{0}\right\|} \in B_{+}$, por lo tanto

$J_{\lambda}\left(u_{n}\right)=\left(\frac{1}{p}-\frac{1}{(q+1)}\right) \int_{[0, \Lambda]} b(x)\left|u_{n}(x)\right|^{q+1}$. 
Por Teorema 4, $L_{0}(\lambda) \subseteq B_{-}, L_{-}(\lambda) \subseteq B_{-} y B_{-} \cap B_{+}=\emptyset$. Así, $\frac{u_{0}}{\left\|u_{0}\right\|} \in L_{+}(\lambda) \cap B_{+}$y por resultados anteriores se obtiene que $\phi_{u_{0}}$ tiene un único mínimo en $t\left(u_{0}\right)$ tal que $t\left(u_{0}\right) u_{0} \in N_{\lambda}^{+}$. Ahora, demostrar que $u_{0}$ está en la Variedad de Nehari. Para eso, supongamos $u_{n} \nrightarrow u_{0}$ en $E_{0}^{\alpha, p}$, entonces

$$
\begin{aligned}
& \int_{[0, \Lambda]}\left(\left|{ }_{0} D_{x}^{\alpha} u_{0}(x)\right|^{p}-\lambda\left|{ }_{0} D_{x}^{\alpha} u_{0}\right|^{p}\right) d x<\lim _{n \rightarrow \infty} \int_{[0, \Lambda]}\left(\left|{ }_{0} D_{x}^{\alpha} u_{n}\right|^{p}-\lambda\left|{ }_{0} D_{x}^{\alpha} u_{n}\right|^{p}\right) d x \\
& \lim _{n \rightarrow \infty} \int_{[0, \Lambda]} b\left|u_{n}(x)\right|^{q+1} d x=\int b(x)\left|u_{0}(x)\right|^{q+1} d x \\
& y \text { por tanto, } t\left(u_{0}\right)=\left[\frac{\int_{[0, \Lambda]} b(x)\left|u_{0}(x)\right|^{q+1} d x}{\int_{[0, \Lambda]}\left(\left.\left|{ }_{0} D_{x}^{\alpha} u_{0}(x)\right|\right|^{p}-\lambda\left|u_{0}(x)\right|^{p}\right) d x}\right]^{\frac{1}{p-(q+1)}}>1 \\
& J_{\lambda}\left(u_{0}\right)=\int_{[0, \Lambda]}\left(\left|{ }_{0} D_{x}^{\alpha} u_{0}(x)\right|^{p}-\lambda\left|{ }_{0} D_{x}^{\alpha} u_{0}\right|^{p}\right) d x-\int_{[0, \Lambda]} b(x)\left|u_{0}(x)\right|^{q+1} d x \\
& <\lim _{n \rightarrow \infty} \int_{[0, \Lambda]}\left(\left|{ }_{0} D_{x}^{\alpha} u_{n}\right|^{p}-\lambda\left|{ }_{0} D_{x}^{\alpha} u_{n}\right|^{p}\right) d x-\int_{[0, \Lambda]} b(x)\left|u_{n}(x)\right|^{q+1} d x=\lim _{n \rightarrow \infty} J_{\lambda}\left(u_{n}\right)
\end{aligned}
$$

Como $\phi_{u_{0}}$ tiene un único mínimo en $t_{u_{0}}$ tal que $t_{u_{0}} u_{0} \in N_{\lambda}^{+}$, se sigue que

$$
\phi_{u_{0}}\left(t_{u_{0}}\right)=J_{\lambda}\left(t\left(u_{0}\right) u_{0}\right)<\phi_{u_{0}}(t), \quad \forall t \in \mathbb{R}^{+},
$$

en particular vale la desigualdad para $t=1$,

$$
J_{\lambda}\left(t_{u_{0}}\left(u_{0}\right) u_{0}\right)<J_{\lambda}\left(u_{0}\right)
$$

Luego por (3.1) y (3.2), se tiene que $J_{\lambda}\left(t\left(u_{0}\right) u_{0}\right)<J_{\lambda}\left(u_{0}\right)<\lim _{n \rightarrow \infty} J_{\lambda}\left(u_{n}\right)=\inf _{u \in N_{\lambda}^{+}} J_{\lambda}(u)$ lo cual es imposible, pues $t_{u_{0}} u_{0} \in N_{\lambda}^{+}$. Por lo tanto $u_{n} \rightarrow u_{0}$ en $E_{0}^{\alpha, p}$ y así $u_{0} \in N_{\lambda}^{+}$, luego, se sigue que $u_{0}$ es un punto minimizador para $J_{\lambda}$ en $N_{\lambda}^{+}$.

Por otro lado $J_{\lambda}(u)=J_{\lambda}(|u|)$ y podemos asumir que $u_{0}$ es no negativo en $[0, \Lambda]$, por lo tanto $J_{\lambda}\left(u_{0}\right)<$ $0, u_{0}$ es un punto mínimo local para $J_{\lambda}$ en $N_{\lambda}^{+}$. Sigue del Lema 5 que $u_{0}$ es un punto crítico de $J_{\lambda}$ y asi es una solución débil del problema de contorno (2.6).

(ii) Sea $\left\{u_{n}\right\} \subseteq N_{\lambda}^{-}$una sucesión minimizante para $J_{\lambda}$ en $N_{\lambda}^{-}$, luego de el Teorema 5, se tiene que $\lim _{n \rightarrow \infty} J_{\lambda}\left(u_{n}\right)=\inf _{u \in N_{\lambda}^{-}} J_{\lambda}(u)>0 ;$ supongamos además que $\left\{u_{n}\right\}$ es no acotada; de modo que, $\left\|u_{n}\right\| \rightarrow$ $\infty$, cuando $n \rightarrow \infty$.

Tomemos $v_{n}=\frac{u_{n}}{\left\|u_{n}\right\|}$, siendo, $\left\{J_{\lambda}\left(u_{n}\right)\right\}$ acotada, se sigue que $\left\{\int_{[0, \Lambda]}\left(\left|{ }_{0} D_{x}^{\alpha} u_{n}(x)\right|^{p}-\lambda\left|u_{n}(x)\right|^{p}\right) d x\right\}$ $y\left\{\int_{[0, \Lambda]}\left(b(x)\left|u_{n}(x)\right|^{q+1}\right) d x\right\}$ son acotadas y por eso

$$
\begin{aligned}
\lim _{n \rightarrow \infty} \int_{[0, \Lambda]}\left(\left|{ }_{0} D_{x}^{\alpha} v_{n}(x)\right|^{p}-\lambda\left|v_{n}(x)\right|^{p}\right) d x & =\lim _{n \rightarrow \infty} \int_{[0, \Lambda]} b(x)\left|v_{n}(x)\right|^{q+1} d x \\
& =\lim _{n \rightarrow \infty} \frac{1}{\left\|u_{n}\right\|^{p}} \int_{[0, \Lambda]} b(x)\left|u_{n}(x)\right|^{q+1} d x=0
\end{aligned}
$$

Como $\left\{v_{n}\right\}$ es acotada, se puede asumir $v_{n} \rightarrow v_{0}$ en $E_{0}^{\alpha, p}$ y $v_{n} \rightarrow v_{0}$ en $L^{p}([0, \Lambda])$ y en $L^{q+1}([0, \Lambda])$, de modo que $\int_{[0, \Lambda]} b(x)\left|v_{0}(x)\right|^{q+1} d x=0$. Si $v_{n} \rightarrow v_{0}$ en $E_{0}^{\alpha, p}$, vemos que $v_{0} \in L_{0}(\lambda) \cap B_{0}$ lo cual es imposible; por la parte $(i)$ del Teorema 4. De allí $v_{n} \nrightarrow v_{0}$ en $E_{0}^{\alpha, p}$ se tiene que $\int_{[0, \Lambda]}\left(\left|{ }_{0} D_{x}^{\alpha} v_{0}(x)\right|^{p}-\right.$ $\left.\lambda\left|v_{0}(x)\right|^{p}\right) d x<\lim _{n \rightarrow \infty} \int_{[0, \Lambda]}\left|{ }_{0} D_{x}^{\alpha} v_{n}(x)\right|^{p}-\lambda\left|v_{n}(x)\right|^{p} d x=0$ Por lo tanto, $v_{0} \neq 0 y \frac{v_{0}}{\left\|v_{0}\right\|} \in$ $L_{-}(\lambda) \cap B_{0}$, lo que es imposible nuevamente. Asi $\left\{u_{n}\right\}$ es acotada y por eso se puede asumir que $u_{n} \rightarrow u_{0}$ en $E_{0}^{\alpha, p}$ y $u_{n} \rightarrow u_{0}$ en $L^{p}([0, \Lambda])$ y en $L^{q+1}([0, \Lambda])$. Supongamos que $u_{n} \nrightarrow u_{0}$ en $E_{0}^{\alpha, p}$. Entonces

$$
\int_{[0, \Lambda]} b(x)\left|u_{0}(x)\right|^{q+1} d x=\lim _{n \rightarrow \infty} \int_{[0, \Lambda]} b(x)\left|u_{n}(x)\right|^{q+1} d x=\left(\frac{1}{p}-\frac{1}{q+1}\right)^{-1} \lim _{n \rightarrow \infty} J_{\lambda}\left(u_{n}\right)<0
$$




$$
\begin{aligned}
\int_{[0, \Lambda]}\left(\left|{ }_{0} D_{x}^{\alpha} u_{0}(x)\right|^{p}-\lambda\left|u_{0}(x)\right|^{p}\right) d x & <\lim _{n \rightarrow \infty} \int_{[0, \Lambda]}\left(\left|{ }_{0} D_{x}^{\alpha} u_{n}(x)\right|^{p}-\lambda\left|u_{n}(x)\right|^{p}\right) d x \\
& =\lim _{n \rightarrow \infty} \int_{[0, \Lambda]} b(x)\left|u_{n}(x)\right|^{q+1} d x=\int b(x)\left|u_{0}(x)\right|^{q+1} d x<0
\end{aligned}
$$

Por lo tanto $\frac{v_{0}}{\left\|v_{0}\right\|} \in L_{-}(\lambda) \cap B_{-}(\lambda)$ y asi $t\left(u_{0}\right) u_{0} \in N_{\lambda}^{-}$, donde

$$
t\left(u_{0}\right)=\left[\frac{\int_{[0, \Lambda]} b(x)\left|u_{0}(x)\right|^{q+1} d x}{\int_{[0, \Lambda]}\left(\left|{ }_{0} D_{x}^{\alpha} u_{0}(x)\right|^{p}-\lambda\left|u_{0}(x)\right|^{p}\right) d x}\right]^{\frac{1}{p-(q+1)}}<1
$$

Además, $t\left(u_{0}\right) u_{n} \rightarrow t\left(u_{0}\right) u_{0}$, pero $t\left(u_{0}\right) u_{n} \nrightarrow t\left(u_{0}\right) u_{0}$ en $E_{0}^{\alpha, p}$, luego $J_{\lambda}\left(t\left(u_{0}\right) u_{0}\right)<\lim _{n \rightarrow \infty} J_{\lambda}\left(t\left(u_{0}\right) u_{n}\right)$. Como el operador $t \rightarrow J_{\lambda}\left(t\left(u_{n}\right)\right.$, alcanza su máximo en $t=1$

$$
\lim _{n \rightarrow \infty} J_{\lambda}\left(t\left(u_{0}\right) u_{0}\right) \leq \lim _{n \rightarrow \infty} J_{\lambda}\left(u_{n}\right)=\inf _{u \in N_{\lambda}^{-}} J_{\lambda}(u)
$$

Por lo tanto, $J_{\lambda}\left(t\left(u_{0}\right) u_{0}\right)<\inf _{u \in N_{\lambda}^{-}} J_{\lambda}(u)$, lo cual es una contradicción. En ese sentido, $u_{n} \rightarrow u_{0}$ en $E_{0}^{\alpha, p}$ y se sigue que $u_{0}$ es un punto minimizador para $J_{\lambda}(u)$ en $N_{\lambda}^{-}$.

Desde que $J_{\lambda}(u)=J_{\lambda}(|u|)$, podemos asumir que $u_{0}$ es no negativo en $[0, \Lambda]$. Dado que $N_{\lambda}^{-}$es cerrado $u_{0}$ es un punto mínimo local para $J_{\lambda}$ en $N_{\lambda}$. Sigue del Lema 5 que $u_{0}$ es un punto crítico de $J_{\lambda}$, y así es una solución débil de problema 1.1.

\section{Conclusiones.}

En este artículo, se discute la existencia de solución débil para un problema no lineal con el operador p-Laplaciano fraccionario, definido en un adecuado espacio de funciones con sus respectivas propiedades. Primero se analiza el comportamiento del funcional asociado al Problema (1.1) para luego utilizar la importante relación entre la Variedad de Nehari y los Fibering Maps y estudiar las propiedades de la Variedad de Nehari que permiten demostrar Teorema 6 que asegura el resutado principal.

\section{Referencias}

[1] Abeliuk Roberto and Howard S. Wheater, Parameter identification of solute transport models for unsaturated soils, Journal of Hydrology, Vol 117:9-18 (1990). Available online 27 March(2003).

[2] Anatoly A. Kilbas, Hari M. Srivastava and Juan J. Trujillo Theory and Applications of Fractional Differential Equations, Elsevier, Amsterdam,ED. Jan Van Mill(2006).

[3] Chen T. and Liu W., Solvability of fractional boundary value problem with p-Laplacian via critical point theory,J. Boundary Value Problems. 75 (2016).

[4] Claudianor Oliveira A. Romildo N. de Lima, Introducão à Teoria dos Pontos Críticos. Unidade Acadêmica de Matematica, Universidade Federal de Campinas Grande. PB/Brasil, Marzo(2018).

[5] Drabek P. and Pohozaev S.I. Positive solutions for the P-Laplacian application of the fibering method Problems, Proceedings of the Royal Socieety of Edinburgh A: Mathematics. Vol127, pags 703 - 726. (1997).

[6] Ervin V. J. and Roop J. P. Variational solution of fractional advection dispersion equations on bounded domains in $R^{d}$, Methods Partial Differential Equations. Vol 23, pag. 256-381 (2007).

[7] Gabriel S., Lau R. and Gabriel C. The dielectric properties of biological tissues: III. parametric models for the dielectric spectrum of tissues,Physics in Medicine and Biology, Vol 42 (1996),no 11, http://stacks.iop.org/0031-9155/41/i=11/a=003, pag. 2271.

[8] Haim Brezis Functional Analysis, Sobolev Spaces and Partial Differential Equations. Springer New York Dordrecht Heidelberg London. pag 58. (2010).

[9] Hartnett M. and Cawley A. M., Mathematical modelling of the effects of marine aquaculture developments on certain water quality parameters, Springer Netherlands, ED. Wrobel, pag. 279-295, (1991), https://doi.org/10.1007/978-94-011-3694-5-20

[10] Hubert H.G.Savenije, Salt intrusion model for high-water slack, low-water slack, and mean tide on spread sheet, Journal of Hydrology, Vol 107(1-4):9-18, (1989). Available online 1 April 2003.

[11] Jiao F. and Zhou Y., Existence of solution for a class of fractional boundary value problems via critical point theory. Comp. Math. Appl., 62, 1181-1199(2011).

[12] Jiao F. and Zhou Y., Existence results for fractional boundary value problem via critical point theory. Inter. Journal of BiF. and Chaos, 22(4), 1-17(2012).

[13] Kenneth E. Bencala, Diane M. McKnight and Gary W. Zellweger, Characterization of transport in an acidic and metal-rich mountain stream based on a lithium tracer injection and simulations of transient storage. Water Resouces Research, Vol 26(5):989-1000, (1990).

[14] Martha E. Londono L., Principio fenomenológico del comportamiento dieléctrico de un hidrogel de alcohol polivinílico - Phenomenological principle dielectrical behaviour of poly (vinyl alcohol) hidrogel,Universidad Nacional de Colombia, Tesis, Medellin(2011).

[15] Matthew F. Causley, Asymptotic and numerical analysis of time-dependent wave propagation in dispersive dielectric media that exhibit fractional relaxation, The State University of New Jersey, (2011).

[16] Meilan Q. and Liquan M., Existence of Weak Solutions for Nonlinear Time-Fractional p-Laplace Problems, Journal of Applied Mathematics. Vol 2014, 9 pages, (2014). 
[17] Nick Laskin, Fractional Schrodinger equation, Physical Review E., Vol 66, art. no. 056108(2002).

[18] Navarrina F., Colominas I., Casteleiro M., Cueto-Felgueroso L., Gómez H., Fe J. and Soage A., Analysis of hydrodynamic and transport phenomena in the R?a de Arousa: a numerical model for high environmental impact estuaries Proceedings of the 8th Congress on Moving Boundary Problems, Vol84, ISSN 1743-3509, (2005), https://www.witpress.com/Secure/elibrary/papers/FSI05/FSI05056FU.pdf

[19] Patyn J., Ledoux E. and Bonne A., Geohydrological research in relation to radioactive waste disposal in an argillaceous formation Journal of Hydrology, Vol 109(3-4): 267-285 (1989).

[20] Podlubny I., Fractional Differential Equations, Academic Press, New York(1999).

[21] Podlubny I., Generalized viscoelastic models: Their fractional equations with solutions, Academic press, New York(1998).

[22] Ross B., Fractional calculus and its aplications: Lecture notas in mathematics. Springer-Verlag, Vol 457, New York(1975).

[23] Ross B., The Development of fractional calculus, Academic Press, Historia mathematica, Vol 4,75-89, New York(1975).

[24] Samko S., Kilbas A. and Marichev O., Fractional Integrals and Derivatives: Theory and Applications, Gordon and Breach Science Publishers. Primera Edición. New York(1993).

[25] Shiessel H., R. Metzler, A. Blumen and T. Nonnenmacher. Generalized viscoelastic models: their fractional equations with solutions. Physics A: Mathematical and General. Vol 28, pp. 6567-6584(1995).

[26] Torres C. Boundary value problem with fractional p-Laplacian operator, DE GRUYTER. Advances in Nonlinear Analysis. Vol 5(2015).

[27] Torres C., Existencia y unicidad de la solución de ecuaciones diferenciales ordinarias de orden fraccionario, Tesis Universidad Nacional de Trujillo,Perú, Pages 59-61(2009).

[28] Torres C. and Nyamoradi N., Existence and multiplicity result for a fractional p-Laplacian equation with combined fractional derivates, Mathematics Subject Classification., 26A33; 35A15; 35B38(2010).

[29] Torres C. and Nyamoradi N., Impulsive fractional boundary value problem with p-Laplacian operator, ED Korean Society for Computational and Applied Mathematics. Pages 22(2016).

[30] Willem Michel Minimax Theorem, Université et Marie Curie. Berlin (1996).

[31] Zhao C Z., Werner M., Taylor S., Chalker P. R., Jones A C and Chun Zhao,Dielectric relaxation of la-doped zirconia caused by annealing ambient, Nanoscale Research Letters. Vol 6(1):48, (2010).

[32] Zhou Y., Basic Theory of Fractional Differential Equations, World Scientifi. Xiangtan University, China. (2014). 